\title{
EXTRACRANIAL METASTASIS OF GLIOBLASTOMA: A RARE CASE
}

\author{
Dessika Rahmawati ${ }^{1}$, Eko Arisetijono Marhaendraputro ${ }^{1}$, Shahdevi Nandar Kurniawan ${ }^{1}$, Andina Wirathmawati ${ }^{1}$ \\ Correspondence: dessika_dr@yahoo.com \\ ${ }^{l}$ Department of Neurology Faculty of Medicine Brawijaya University, Malang, Indonesia.
}

\author{
Article History: \\ Received: December 10, 2018 \\ Accepted: January 7, 2019 \\ Published: January 1, 2019

\section{Cite this as:} \\ Rahmawati D, Marhaendraputro \\ EA, Kurniawan SN, \\ Wirathmawati A. Extracranial \\ metastasis of glioblastoma: a \\ rare case. Malang Neurology \\ Journal; 2019.5:51-54. DOI: \\ http://dx.doi.org/10.21776/ub.mnj \\ .2019.005.01.10
}

\section{ABSTRACT}

Glioblastoma is the most common primary malignant tumor in the neural tissue and a highly aggressive primary tumor in human. It involves glial cells in $52 \%$ of functional brain tissue cases and $20 \%$ of all intracranial tumors. Extra cranial metastasis of Glioblastoma is very rare. Metastasis outside CNS was uncommon in GBM but it could occur with frequency $0.2 \%$ and can spread in head and neck sites. The mechanism of this spread is not understood yet. The extracranial spread routes were speculated on the direct lymphatic connections, the venous system and the adjacent structure like dura and bone. We present a case of female patient with a known glioblastoma who had an extracranial metastasis on her left neck with complete Radiotherapy n Chemotherapy and survive more than 2 years.

Keywords: Glioblastoma, Extracranial metastasis, Neck mass, Rare case

\section{Introduction}

Glioblastoma is one of the most malignant neoplasms with high grade undifferentiated glial neoplasm in human and it is known as a malignant neoplasm in cerebral hemisphere. ${ }^{1}$ The incidence of glioblastoma is 3.19/100000 patient per year. Mortality was high with death commonly occurred after 18 months from diagnosis and with median survival time 12 months. ${ }^{2}$ Metastasis outside CNS was uncommon in Glioblastoma (GBM) but it could occur with frequency $0.2 \%$ and can spread in head and neck sites. The underlying mechanism of extracranial metastasis is not known. The extracranial spread routes were speculated on the direct lymphatic connections, the venous system and the adjacent structure like dura and bone. ${ }^{12}$ In this case we present a GBM patient with extracranial metastasis to collar region who lived more than 18 month.

\section{Case Report}

A 37-year-old female admitted to hospital with a chronic progressive right hemiparesis since one year before admission. She also had right hemiparesthesia, left facial paresis, and disartria. At the moment, she also has motoric aphasia, a protruding right eye and blindness in both eyes, and a mass in the left neck.

The initial symptoms were intermittent headache in the left side, particularly in the morning, but with no vomiting, which started about 2 years before admission. The patient also had general tonic clonic seizure 3 years before admission.

The patient had a head CT-Scan two years ago and was diagnosed with a brain tumor. In the past medical history, she had 2 surgeries in the left frontoparietal region. The first surgery was in a secondary hospital one year ago and the second one in Dr. Saiful Anwar General Hospital (tertiary hospital) three months before current admission. The patient had received Radiotherapy for 6 weeks and a Temozolamide chemotherapy for 6 months.

At admission, Head and neck examination found a mass in left collar region (dimension 10x15x3 cm), solid, with clear edge and fixated.

Head Magnetic Resonance Imaging (MRI) found a cystic encephalomalacia in the left frontotemporal lobe with a solid mass within, possibly a residual mass, and a moderate communicant hydrocephalus.

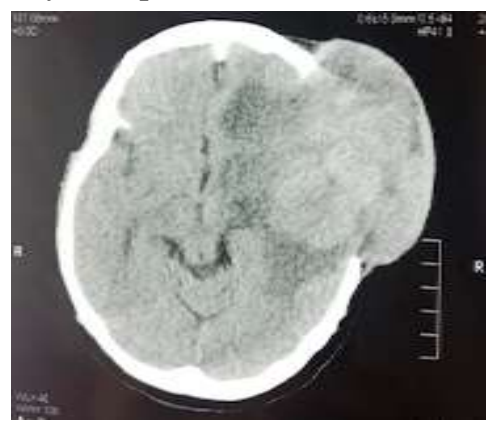

(A)

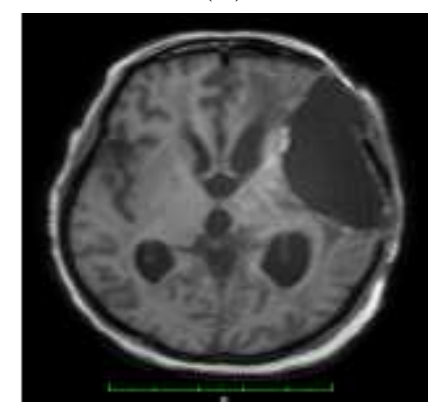

(B) 


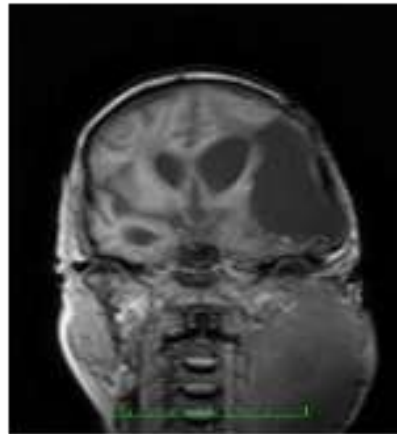

(C)

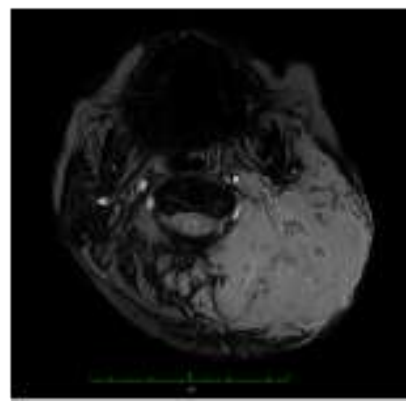

(D)

Figure 1. (A) Head CT Scan 1 year after first surgery, the tumor was relapsing, (B) Head MRI post second surgery shows cystic encephalomalacia, (C) \& (D) Head and Neck MRI 2 months after second surgery show mass in the left collar region.

The mass in the posterior left collar region is suggestive of a malignancy. Pathological examination of brain tissue revealed proliferating tumor cells with variable shape and size, pleomorphic, hyperchromatic, and partially prominent nucleolus, mitosis 30/10 HPF, giant cells were found as well as endothelial proliferation and necrosis; the conclusion was Glioblastoma (WHO grade IV). Fine Needle Aspiration Biopsy (FNAB) of the collar mass found small round tumor cells which is usually found in blastomas. The neck mass biopsy and histopathology result indicated a metastatic of GBM. Immunohistochemical staining for glial and and epithelial markers revealed positive glial fibrillary acidic protein (GFAP) and neuron-spesific enolase (NSE), and therefore a metastatic Glioblastoma to the left collar region was established.

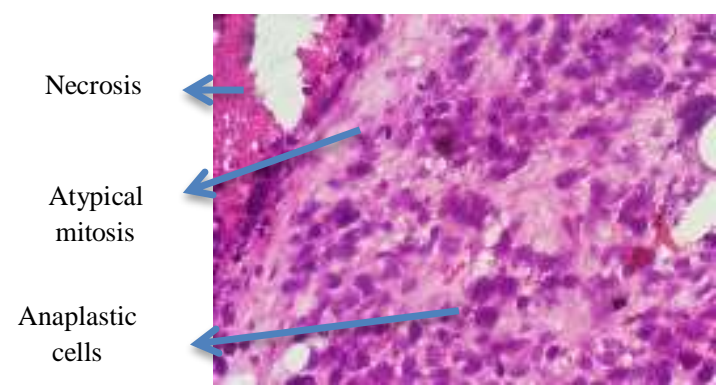

(A)

Anaplastic

cells and

erithrocyte

extravasation

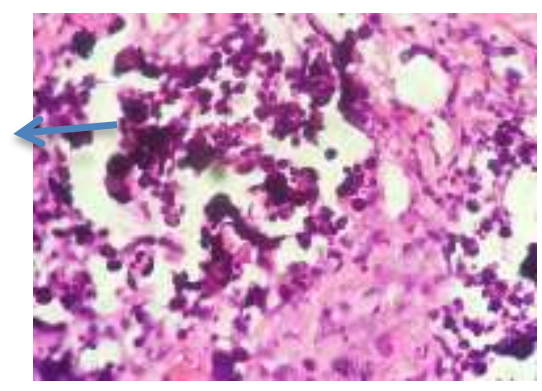

(B)

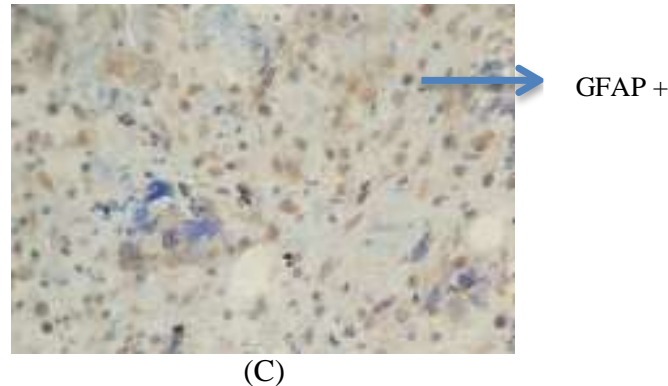

Figure 2. (A) Haematoxilin Eosis stain supporting Glioblastoma (WHO grade IV), (B) Untypical neoplasm from neck mass biopsy, suggest to metastasis from GBM, (C) Immunohistochemical staning result with possitive GFAP.

Excision of tumor mass in the collar region was planned and Temozolamide chemotherapy will be resumed for 6 months. Inspite of the aggressive treatment for the tumor, the patient was deteriorating and passed away 3 months later.

\section{Discussion}

Glioblastoma is aggressive diffuse forms of glial tumor, representing approximately $25 \%$ of primary Central Nervous system (CNS) tumours in adults. The tumour is associated with a strong tendency for local invasion, with a high rate of local relapse, severe disability and a high risk of death. ${ }^{14}$ However, extracranial spread of GBM is very rare, with reported incidence of $0,2 \%{ }^{15}$

Glioblastoma metastasis mechanism, espescially extracranial, although poorly understood has been suggested by cervio et al in three possible mechanism 1) lymphatic drainage of CSF to extraneural tissue, 2) venous invasion via leptomeningeal tissue, 3) direct invasion through dura and bone along VP drain. ${ }^{13}$ Another possible mechanism was quiet simple, is based on surgical intervention which breaks the blood brain barrier and opening on the brain vessel. Although surgery has markedly increased the incidence of extracranial metastasis of glioblastoma, some cases were without history of surgery. Common sites for glioblastoma metastasis was lung and pleura $(59.7 \%)^{13}$, lymphnode $(51.4 \%)$, bone $(30.5 \%)$, liver $(22.2 \%)$ and other sites like cervical region (neck). In our case the patient had several times surgical intervention which might increase of extracranial metastase.

It is important to note that extracranial metastasis from GBM is due to highly local nature. There are several hypotheses that have been suggested to explain the rarity of extraneural spread of glioblastoma. The first hypothesis was suggested based on poor survivals of patient. Second hypotheses was based on the concept of capillary basement membrane barrier which play role as physical barrier. Other hypotheses include spread from extracellular matrix through dura and lymphatics of the brain. ${ }^{1}$ Another assumption is, the cellular environment of CNS has prevented natural metastastic process which mainly due to components of extracellular matrix of the brain especially in blood vessels that lacks of collagen and fibronectin which occur at hyperplastic blood vessel, interestingly, extracellular space components mainly fulfilled by hyaluronic acid and glycosaminoglycans which can spread tumor cells into adjacent tissue. This property made hematogenous metastasis really rare to occur. 
The extracranial metastasis of glioblastoma could be related with the following factors: (1) Age during diagnosis, which Piccirilli et al. $^{5}$ reported on 128 cases of glioblastoma extracranial metastases in 2008. The mean age of the patients at presentation was 40 years; while other patients with glioblastoma was 54 years. $^{6}$ The young glioblastoma patients have higher potential for extracranial metastasis event. (2) Lifespan: with an accurate of treatment and better diagnostic tools, patients with glioblastoma have increased their survival time, which lead to the potential for increasing extracranial metastases event. Moreover, metaanalysis supported the hypotheses that increasing survival of patients expand the probability of glioblastoma cells spreading through hematogenous and lymphatic systems. ${ }^{7}$ (3) Surgical treatment: most patients $(96 \%)$ reported with extracranial metastases had prior cranial surgery. ${ }^{8}$ Surgery may lead to dural damage and potentially facilitate tumor cells crossing the dura mater, which is the most important barrier. Primarily, the operation should strictly adhere to the tumor-free technique and ensure completeness of cerebral dura mater for reducing the occurrence of extracranial metastasis event ${ }^{10}$. (4) Spontaneous metastasis without surgery. Even though surgery is one of risk factor for occurrence of extracranial metastasis, for some cases the metastasis can occur without any surgery.

Extracranial metastasis could be related to tumor chemoradiotherapy. Tuettenberg et $a l .{ }^{12}$ reported the mechanism of glioma angiogenesis inhibition and treatment with radiotherapy and chemotherapy, where tumor angiogenesis and primary tumor growth was inhibited, but tumor cell invasion and proliferation was increased to the brain tissue. Gamma rays interact with body molecules causing damage to DNA and subsequently apoptosis. ${ }^{11}$

To establishing criteria of extracranial metastasis should be met: 1) From histologically metastatic lesion identified as CNS tumor; 2) clinical history must indicate CNS tumor as initial neoplasm; 3) complete autopsy must be performed to exclude the possibility of other primary tumor; 4) morphologic features of the primary lesion and metastatic lesion must be identical. In this case, our patient fulfilled this criteria.

The presence of extraneural metastases does not significantly affect the already dismal prognosis of recurrent GBM, but appropriate therapy may increase the quality of life. Detection of extracranial metastases is important by itself for prediction of short survival; only palliative therapy is available when they cause symptoms.

Our patient, has been diagnosed with GBM for over 2 years and had two surgical interventions followed by radiotherapy with a dose of $60 \mathrm{~Gy}$ and recent temozolamide chemotherapy. The mass was found in the left side of the collar region was confirmed with histopathological and immunohistochemical results, in accordance with extracranial metastasis of Glioblastoma. Possible mechanisms of metastasis from glioblastoma have not been ascertained yet, either through hematogenous or lymphatogenous or due to several craniotomy surgeries that have been carried out which can lead to direct invasion. This patient survives more than 2.5 years, which is longer than most glioblastoma patients, so that it is suspected that metastasis is found to be associated with a longer duration of life in these patients, in which the average patient has died less than 2 years.

\section{Conclusion}

A patient with Glioblastoma of the brain had extracranial metastasis on collar region. The underlying mechanism of extracranial metastasis is exceptionally unclear. This case presents the possibility of extracranial metastasis glioblastoma, the main risk factor in this patient was the recurrent surgeries and a long lifespan.

\section{Acknowledgement}

The author wishes to acknowledge dr. Diah Prabawati Retnani, Sp.PA (K) for assistance in pathological examination, Prof. Dr.dr. Yuyun Yueniwati P. W., M.Kes, Sp.Rad (K) and dr. Achmad Bayhaqi Nasir Aslam, Sp.Rad for radiological examination, and the Neurosurgeon dr. Donny Wisnu Wardhana, Sp.BS for their contributions in thorough management of the case being reported.

\section{References}

1. Haroon $\mathrm{S}$ and Faridi NF. Cervical metatstatic glioblastoma multiforme. Journal of the College of Physicians and Surgeons Pakistan; 2013.23(2):160-1. DOI: 02.2013/JCPSP.160161

2. Visser O, Ardanaz E, Botta L, Sant M, Tavilla A, Minicozzi P, Survival of adults with primary malignant brain tumours in europe; results of the eurocare-5 study. Eurocare-5 Working Group: Eur J Cancer; 2015.51(15):2231-2241. DOI: 10.1016/j.ejca.2015.07.032

3. Hoffman HJ, Duffner PK. Extraneural metastases of central nervous system tumors. Cancer; 1985.1;56(7 Suppl):1778-82.

https://www.ncbi.nlm.nih.gov/pubmed/4027909

4. Catherine C, Park, et.ala. Systemic Metastasis in glioblastoma may represent the emergence of neoplastic subclones. J Neuropathol Exp Neurol; 2000.59:1044-50

5. Piccirilli M, Brunetto GM, Rocchi G, Giangaspero F, Salvati M. Extra central nervous system metastases from cerebral glioblastoma multiforme in elderly patients. clinico-pathological remarks on our series of seven cases and critical review of the literature. Tumori; 2008.94(1):40-51. PubMed: https://www.ncbi.nlm.nih.gov/pubmed/18468334

6. Polley MY, Lamborn KR, Chang SM, Butowski N, Clarke JL, Prados M. Conditional probability of survival in patients with newly diagnosed glioblastoma. J Clin Oncol; 2011.1;29(31):4175-80. DOI: $10.1200 / J C O .2010 .32 .4343$

7. Anghileri E, Castiglione M, Nunziata R, Boffano C, Nazzi V, Acerbi F, et al. Extraneural metastases in glioblastoma patients: two cases with ykl-40-positive glioblastomas and a meta-analysis of the literature. Neurosurg Rev; 2016.39(1):37-45; discussion 45-6. DOI: $10.1007 / \mathrm{s} 10143-015-0656-9$

8. Huang P, Allam A, Taghian A, Freeman J, Duffy M, Suit HD. Growth and metastatic behavior of five human glioblastomas compared with nine other histological types of human tumor xenografts in scid mice. J Neurosurg; 1995.83(2):308-15. PubMed: https://www.ncbi.nlm.nih.gov/pubmed/7616277 
9. Schweitzer T, Vince GH, Herbold C, Roosen K, Tonn JC. Extraneural metastases of primary brain tumors. J Neurooncol; 2001.53(2):107-14. PubMed: https://www.ncbi.nlm.nih.gov/pubmed/11716064

10. Zustovich F, Della Puppa A, Scienza R, Anselmi P, Furlan C, Cartei G. Metastatic oligodendrogliomas: a review of the literature and case report. Acta Neurochir (Wien); 2008.150(7):699-702; discussion 702-3. DOI: $10.1007 / \mathrm{s} 00701-008-1507-\mathrm{z}$

11. Alam ABN, Rahayu M, Islani AZS. Effect of the radiation of gamma rays on caspase- 3 expression in rattus norvegicus wistar male variant brain cell with immunohistochemistry method. Malang Neurology Journal; 2015.1:72-9.

DOI: http://dx.doi.org/10.21776/ub.mnj.2015.001.02.5

12. Tuettenberg J, Grobholz R, Korn T, Wenz F, Erber R, Vajkoczy P. Continuous low-dose chemotherapy plus inhibition of cyclooxygenase- 2 as an antiangiogenic therapy of glioblastoma multiforme. J Cancer Res Clin Oncol; 2005.131(1):31-40. DOI: 10.1007/s00432-0040620-5

13. Cervio A, Piedimonte F, Salaberry J, Alcorta SC, Salvat J, Diez B, et al. Bone metastases from secondary glioblastoma multiforme: a case report. J Neurooncol; 2001.52(2):141-8. PubMed: https://www.ncbi.nlm.nih.gov/pubmed/11508813

14. Kaye A, Laws Jr E. Brain tumours. Philadelphia: Churchill Living-stone; 2001

15. Hsu E, Keene D, Ventureyra E, et al. Bone marrow metastasis in astrocitic glioma. J Neurooncol; 1998.37:285-93. PubMed: 